Pacific Journal of Mathematics

DISCONTINUOUS CHARACTERS AND SUBGROUPS OF

(1) 


\title{
DISCONTINUOUS CHARACTERS AND SUBGROUPS OF FINITE INDEX
}

\author{
H. LEROY PETERSON
}

For brevity's sake, a subgroup of finite index is called "large." A discontinuous torsion character is (clearly) one whose kernel is a large nonopen subgroup. Compact Abelian groups (and certain other $L C A$ groups) have the same number (either none or infinitely many) of large nonopen subgroups and discontinuous torsion characters. Locally compact Abelian groups of which all large subgroups are open include connected, locally connected, and monothetic (possibly totally disconnected) groups. Contrariwise, there are locally compact groups $G$ which have as many as $2^{|G|}$ large nonopen subgroups. These include nondiscrete torsion Abelian groups of bounded order and all totally disconnected, nonmetrizable, compact groups.

1.1. Notation and discussion. In what follows, everything referred to as a locally compact (or compact) group will be assumed to be Hausdorff and nondiscrete, except when it is necessary to refer to a discrete group or a nondiscrete finite group in the course of a proof. If $G$ is a group, $G_{d}$ denotes $G$ with the discrete topology. If $n$ is a positive integer, $G^{(n)}=\left\{x^{n}: x \in G\right\}$. The symbol $e$ will universally denote the identity of a group. For two topological groups $G_{1}$ and $G_{2}, " G_{1} \cong G_{2}$ " means that $G_{1}$ and $G_{2}$ are topologically isomorphic. The component of $e$ in a topological group $G$ is denoted by $C_{G}$. The weight of $G$, denoted by $w(G)$, is the smallest cardinal number for a basis for the topology of $G$. The symbol $T$ will denote the circle group (of complex numbers with modulus one); a character on an Abelian group $G$ is a homomorphism (continuous or not) from $G$ into $T$. If $X$ is a set, $|X|$ denotes the cardinal number of $T$.

Vol. I of the treatise of Hewitt and Ross [3] will be used as an encyclopedic reference; however, some elementary facts to be found in any text on topological groups will be assumed and used without comment.

It is evident that the closure of a large subgroup (as defined in the synopsis) of any topological group is always a large open subgroup, and that every open subgroup of a compact group is large. As stated in the synopsis, the kernel of a discontinuous character of finite order is a large nonopen subgroup. More generally, a large subgroup of an Abelian topological group is nonopen if and only if it is contained in the kernel of a discontinuous torsion character. (Equivalently, a finite Abelian topological group has discontinuous characters if and only if the group is not discrete.) 
The first few results of the sequel have to do with necessary conditions for the existence of large nonopen subgroups. A fundamental relationship between large nonopen subgroups and discontinuous torsion characters is established in Theorem 1.7; the remainder of this section is concerned with consequences of this result.

Lemma 1.2. Let $G$ be a locally compact Abelian or compact group. Let $L$ be a large nonopen subgroup of $G$ and let $C=C_{G}$. Then $C \subset L$ and $C$ is not open.

Proof. By the results in [7], or by 24.24 of [3] (whichever is appropriate) $C$ is contained in a divisible subgroup $H$ of $G$. Let $n=[G: L]$; then $x^{n !} \in L$ for all $x \in G$, and thus $C \subset H \subset L$. Since $L$ has void interior, $C$ is not open.

CoRollary 1.3. Let $G$ be a group as in 1.2. If $G$ has only finitely many open subgroups, every large subgroup of $G$ is open.

Proof. $C$ is open in this case.

[Note: (1.10.3), below, shows that 1.3 need not be true if $G$ is not Hausdorff.]

THEOREM 1.4. Every large subgroup of a compact monothetic group is open.

Proof. Let $L$ be a large subgroup of $G$, let $n=[G: L]$, let $J=$ $G^{(n)}$, and let $H$ be a dense cyclic subgroup of $G$. We have $J \subset L$, and $J$ is compact and therefore closed. Now $H J / J$ is a dense subgroup of $G / J$, but $H J / J$ is cyclic and each of its elements has order $\leqq n$; thus $|H J / J| \leqq n$. Since $G / J$ is a Hausdorff space with a finite dense subset, it is finite. Thus $J$ is open and since $J \subset L, L$ is open also.

COROLLARY 1.5. A character on a compact totally disconnected monothetic group is continuous if and only if it is of finite order.

[Note: Such groups are characterized in [3], 10.1-10.6 and 25.16.]

Proof. The theorem implies that every character of finite order is continuous. The converse is contained in 24.26 of [3].

Lemma 1.6. Let $A$ and $B$ be two sets, at least one of which is infinite. Let $\mathscr{A}$ be a collection of finite subsets of $A$. Then: 
(1) If $|B| \leqq|\mathscr{A}|,|B| \leqq|A|$.

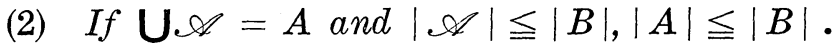

Proof. A simple exercise.

THeORem 1.7. Let $G$ be a locally compact Abelian group, $D$ the set of discontinuous torsion characters on $G$, and $\mathscr{L}$ the family of large nonopen subgroups of $G$. Then, if either $D$ or $\mathscr{L}$ is infinite, $|D|=|\mathscr{L}|$.

Proof. Let $\Gamma$ denote the group of characters on $G$, and $K$ (respectively, $K_{c}$ ) denote the group of (continuous) torsion characters on $G$. We may consider $\Gamma$ to be the dual group of $G_{d}$, and $K$ its torsion subgroup. Similarly, $K_{c}$ is the torsion subgroup of the dual group of $G$.

We may assume that $\mathscr{L} \neq \varnothing \neq D$. Let $\phi$ be any member of $K$, and let $S(\phi)$ denote the set of all members of $K$ which have the same kernel as $\phi$. Clearly, if $\phi \in D$, then $S(\phi) \subset D$. Also, $\psi(G)=\phi(G)$ for all $\psi \in S(\phi)$, since both groups are finite subgroups of $T$ and have the same order. For each $\psi \in S(\phi)$, there is an automorphism $\gamma$ of $\phi(G)$ such that $\psi=\gamma \circ \phi$, namely $\gamma(t)=\psi\left(\phi^{-1}(t)\right)(t \in \phi(G))$. But $\phi(G)$ has only finitely many automorphisms; thus $S(\phi)$ is finite. Let $\mathscr{S}=$ $\{S(\phi): \phi \in D\}$. Then $\cup \mathscr{S}=D$ and $|\mathscr{S}| \leqq|\mathscr{L}|$. By 1.6, if either $\mathscr{L}$ or $D$ is infinite, $|D| \leqq|\mathscr{L}|$, and we may assume henceforth that $\mathscr{L}$ is infinite. Since $D=K \cap\left(K_{c}\right)^{\prime} \neq \varnothing, D$ contains a coset of $K_{c}$; thus $\left|K_{c}\right| \leqq|D| \leqq|\mathscr{L}|$. If $|\mathscr{L}| \leqq\left|K_{c}\right|$, we are through. Suppose contrariwise that $\left|K_{c}\right|<|\mathscr{L}|$. For any large subgroup $H$, let $A(H)=$ $\{\psi \in \Gamma: \psi(H)=1\}$; clearly $A(H)$ is a finite subgroup of $K$, and $A(H) \subset K_{c}$ if and only if $H$ is open. Further, $H \rightarrow A(H)$ is a one-toone correspondence, since $H=\{x \in G: \psi(x)=1$ for all $\psi \in A(H)$. $\} \quad$ (See (24.10) of [3].) Let

$$
\mathscr{A}=\left\{K_{c} \cap A(H): H \in \mathscr{L}\right\},
$$

a collection of finite subgroups of $K_{c}$ with $|\mathscr{A}| \leqq|\mathscr{L}|$. If $\mathscr{A}$ is finite, $|\mathscr{A}|<|\mathscr{L}|$ (trivially). If $\mathscr{A}$ is infinite, then $K_{c}$ is infinite and thus $|\mathscr{A}| \leqq\left|K_{c}\right|<|\mathscr{L}|$. In either case, there exists a subcollection $\mathscr{H}$ of $\mathscr{L}$ such that $|\mathscr{L}|=|\mathscr{L}|$ and $K_{c} \cap A\left(H_{1}\right)=$ $K_{c} \cap A\left(H_{2}\right)$ for all $H_{1}, H_{2} \in \mathscr{C}$. But if $H_{1} \neq H_{2}, A\left(H_{1}\right) \neq A\left(H_{2}\right)$, and therefore $D \cap\left(A\left(H_{1}\right)\right) \neq D \cap\left(A\left(H_{2}\right)\right)$ (since $\left.D=K \cap\left(K_{c}\right)^{\prime}\right)$. Consequently, $\mathscr{B}=\{D \cap A(H): H \in \mathscr{C l}\}$ has the same cardinality as $\mathscr{C l}$; but then $|\mathscr{L}|=|\mathscr{C}|=|\mathscr{B}|$, thus $|\mathscr{L}| \leqq|D|$.

THeorem 1.8. Let $G$ be a compact Abelian group, and $\mathscr{Q}$ the family of open subgroups of $G$. If $D \neq \varnothing$, then $|D| \geqq|\mathscr{C}|$. Thus $G$ has either infinitely many large nonopen subgroups, or none. 
Proof. Suppose $D \neq \varnothing$, so that $\mathscr{L} \neq \varnothing$ and (by 1.3) $\mathscr{U}$ is infinite. Now (as in the proof of 1.7) $|D| \geqq\left|K_{c}\right|$ and the mapping $H \rightarrow A(H)$ is one-to-one from $\mathscr{U}$ onto the collection of all finite subgroups of $K_{c}$; thus $|\mathscr{U}| \leqq\left|K_{c}\right| \leqq|D|$ and (by 1.7) $|D|=|\mathscr{L}|$.

LEMmA 1.9. Let $G$ be a locally compact Abelian group with a large nonopen subgroup. Then every open subgroup of $G$ has a large nonopen subgroup. If $G$ is compact and totally disconnected, every neighborhood of the identity contains a large nonopen subgroup of $G$.

Proof. Let $U \in \mathscr{L}$ and $L \in \mathscr{L}$. Then $[U: U \cap L]=|\{u L: u \in U\}| \leqq$ [G:L]. $U \cap L$ is not open since $L$ has void interior. If $G$ is compact and totally disconnected, every neighborhood of $e$ contains a (large) open subgroup of $G$. If $U$ is one such subgroup, $[G: U \cap L]=[G: U]$. $[U: U \cap L] \leqq[G: U] \cdot[G: L]$. Thus $U \cap L$ is a large nonopen subgroup of $G$.

THEOREM 1.10. Let $G$ be an infinite compact Abelian torsion group.

Then

(1) $G$ has $2^{|G|}$ discontinuous torsion characters (and, therefore, $2^{|\epsilon|}$ large nonopen subgroups);

(2) Every neighborhood of the identity contains a large nonopen subgroup of $G$.

(3) Every proper dense subgroup of $G$ is contained in a large proper subgroup of $G$.

Proof. (1) By 25.9 of [3], $G^{(n)}=\{e\}$ for some integer $n$, so every character on $G$ is of finite order. Now the characters on $G$ form a compact group (namely, the dual group of $G_{d}$ ) whose weight is $|G|$ and whose cardinality is therefore $2^{|\epsilon|}$. (See 24.15 and 24.47 of [3].) But $\left|K_{c}\right|=w(G)$, where $|G|=2^{w(G)}>W(G)$; thus $G$ has $2^{|G|}$ discontinuous characters. (The rest of the statement follows from 1.7.)

(2) By 24.21 of [3], $G$ is totally disconnected, so Lemma 1.9 applies.

(3) If $H$ is a proper dense subgroup of $G$, there is a character $\psi$ on $G$ such that $\psi(H)=1$ and $\psi(G) \neq 1$. The kernel of $\psi$ is a large proper subgroup containing $H$.

Note: Statement (3) is a generalization of an example, communicated by K. A. Ross, in which $G=\{0,1\}^{A}$. (Ross' proof is as follows: Choose $x \in G \cap H^{\prime}$ and let $\psi$ be the homomorphism of $H \oplus[x]$ into $T$ such that $\psi(H)=1$ and $\psi(x)=-1$. Extend $\psi$ to a character of order 2 on $G$.) 
COROLLARY 1.11. Let $G$ be a locally compact Abelian group of bounded order. Then statements (1) and (3) of 1.10 are true.

Proof. As before (in 1.10.1), $G$ has $2^{|G|}$ characters, all of finite order. Since $G$ is totally disconnected, it has a compact open subgroup. (See 24.21 and 7.7 of [3].) This subgroup has a discontinuous character which may be extended to a character on $G$. Thus $G$ has $2^{|G|}$ discontinuous characters. (Using the terminology of 1.7, we have $D \neq \varnothing$. Thus $|D| \geqslant\left|K_{c}\right|$, and since $\left|D \cup K_{c}\right|=2^{|G|},|D|=2^{|G|}$.) The proof of statement (3) can be applied just as in 1.10.

Note: For an example of a locally compact group which has exactly the same number of open and nonopen large subgroups, let $G=H \times H_{d}$ where $H$ is a compact Abelian torsion group. Here, $G$ and $G_{d}$ have the same number of continuous characters, namely $2^{|c|}$ of each.

\section{Large nonopen subgroups of compact groups.}

Discussion 2.1. Let $\left\{V_{\alpha}: \alpha \in A\right\}$ be an infinite collection of open subgroups of a locally compact group $G$. Let $\mathscr{F}$ be a free ultrafilter on $A$. We write

$$
L\left(\mathscr{F}, V_{\alpha}\right)=\bigcup_{F \in \mathscr{F}} \bigcap_{a \in F} V_{\alpha} \text {. }
$$

In this section, we describe sufficient conditions under which $L\left(\mathscr{F}, V_{\alpha}\right)$ is a large nonopen subgroup of $G$.

The reader may recall that a free ultrafilter is a maximal filter with no fixed point (see [2], 2.2-2.6 and 12.2). Thus if $\mathscr{F}$ is a free ultrafilter on $A$, and $\left\{F_{1}, \cdots, F_{n}\right\}$ is a finite partition of $A$, exactly one of the cells $F_{j}$ is in $\mathscr{F}$. Also, no finite subset of $A$ is a member of $\mathscr{F}$.

LeMma 2.2. Let $G$ be a compact group, $\mathscr{U}$ and $\mathscr{Y}$ infinite collections of open subgroups of $G$, with $\mathscr{U} \subset \mathscr{V}$ and $\cap \mathscr{U}=\bigcap \mathscr{Y}$. Then $|\mathscr{U}|=|\mathscr{V}|$.

Proof. We may assume that $\mathscr{U}$ is closed under finite intersections. It follows that each member of $\mathscr{V}$ is the union of (finitely many) cosets of a member of $\mathscr{U}$.

Lemma 2.3. Suppose $H$ is large open subgroup of a locally compact group $G, J$ is normal subgroup of $H$, and $L / J$ is a large nonopen subgroup of $H / J$ (where $J \subset L \subset H$ ). Then $L$ is a large nonopen subgroup of $G$. 
Proof. We have

$$
[G: L]=[G: H][H: L]=[G: H][H / J: H / L] \text {; }
$$

i.e., $L$ is large in $G$. If $L$ is open, $L$ is locally compact. But then $L / J$ is locally compact, which is impossible since $L / J$ is not closed in $H / J$. ([3], 5.22 and 5.11).

THEOREM 2.4. Let $G$ be a compact group. Suppose there is an infinite collection $\mathscr{Q}$ of open normal subgroups of $G$ and a fixed integer $n$ such that $|G / U| \leqq n$ for all $U \in \mathscr{U}$. Then $G$ has $2^{2 r}$ large nonopen subgroups, where $r=|\mathscr{Q}|$.

Proof. Case (1). Suppose $G=S^{A}$ where $S$ is a finite group and $A$ is an infinite set. For $\alpha \in A$, define $V_{\alpha}=\left\{x \in G: x_{\alpha}=e\right\}$. (Here, $\mathscr{U}=\left\{V_{\alpha}: \alpha \in A\right\}, n=|S|$, and $\left.r=|A|.\right)$ Let $\mathscr{F}$ be any free ultrafilter on $A$, and $L=L\left(\mathscr{F}, V_{\alpha}\right)$. One sees that $L$ is a dense normal subgroup of $G$, via a minor generalization of the familiar proposition that

$$
\left\{x \in G: x_{\alpha}=e \text { for all but finitely many } \alpha\right\}
$$

is a dense normal subgroup. Now, given any $x \in G$, there exists exactly one element $s(x)$ of $S$ such that $\left\{\alpha \in A: x_{\alpha}=s(x)\right\}$ is a member of $\mathscr{F}$. Thus $[G: L]=|S|$; in fact, $(G / L)_{d} \cong S$. This proves that $L$ is a large, but proper, subgroup of $G$, and since $L$ is dense it is not open. Now suppose $\mathscr{F}_{1}$ and $\mathscr{F}_{2}$ are distinct ultrafilters on $A$. We will show that $L\left(\mathscr{F}_{1}, V_{\alpha}\right) \neq L\left(\mathscr{F}_{2}, V_{\alpha}\right)$. For there is a subset $X$ of $A$ such that $X \in \mathscr{F}_{1}$ and $A \cap X^{\prime} \in \mathscr{F}_{2}$; let $e \neq s \in S$, and define

$$
x_{\alpha}=\left\{\begin{array}{l}
e, \alpha \in X \\
s, \alpha \in A \cap X^{\prime} .
\end{array}\right.
$$

Then $\left(x_{\alpha}: \alpha \in A\right)$ is in $L\left(\mathscr{F}_{1}, V_{\alpha}\right)$ but not $L\left(\mathscr{F}_{2}, V_{\alpha}\right)$. According to [2], $(9 \mathrm{~F}), A$ has $2^{2 r}$ ultrafilters, of which only $r$ are not free. Thus $G$ has $2^{2^{r}}$ large nonopen subgroups.

Case (2). Suppose all the quotient groups $G / U$ are simple and isomorphic to each other and $\cap \mathscr{U}=e$. We can then choose a subfamily $\left\{U_{\alpha}: \alpha \in A\right\}$ of $\mathscr{C}$ such that

$$
G \cong \prod_{a \in A} G / U_{\alpha}
$$

via the mapping $g \rightarrow\left(g U_{\alpha}: \alpha \in A\right)$ (as in Varopoulos [8], $\S 4$, Theorem 2). By 2.2 (above), $|A|=|\mathscr{C}|$. Case (1) now applies. $G$ has $2^{2 r}$ large nonopen subgroups of the form $L\left(\mathscr{F}, U_{\alpha}\right)$. 
Case (3). In general, we may choose a subfamily $\left\{U_{\alpha}: \alpha \in A_{0}\right\}$ of $\mathscr{U}$, with $\left|A_{0}\right|=|\mathscr{C}|$ and $U_{\alpha} \neq U_{\beta}$ for $\alpha \neq \beta$, and such that all of the quotient groups $G / U_{\alpha}$ are isomorphic; this is possible since there are only finitely many groups (to within isomorphism) of order $\leqq n$. Let

$$
U_{\alpha}=G_{\alpha}^{m} \subset G_{\alpha}^{m-1} \subset \cdots \subset G_{\alpha}^{0}=G
$$

be a maximal subnormal series (modulo $U_{\alpha}$ ), chosen so that $m$ is the same for each $\alpha$, and so that

$$
G_{\alpha}^{j-1} / G_{\alpha}^{j} \cong G_{\beta}^{j-1} / G_{\beta}^{j}
$$

for all $\alpha, \beta \in A_{0}$ and each $j(1 \leqq j \leqq m)$. Let $k$ be the smallest integer with the property that there are $r$ distinct subgroups $G_{\alpha}^{k}$. Let $V_{\alpha}=G_{\alpha}^{k}$ for each $\alpha \in A_{0}$. We now have an open subgroup $H$ of $G$ and a subset $A_{1}$ of $A_{0}$, such that $\left|A_{1}\right|=r, H=G_{\alpha}^{k-1}$ for all $\alpha \in A_{1}$, and $V_{\alpha} \neq V_{\beta}$ for $\alpha \neq \beta$. Let $J=\bigcap_{\alpha \in A_{1}} V_{\alpha}$. Recall that each $V_{\alpha}$ is an open maximal normal subgroup of $H$, and all of the groups $H / V_{\alpha}$ are isomorphic. Case (2) applies to the group $H / J$, which therefore has $2^{2^{r}}$ large nonopen subgroups. Since $H$ is open (and therefore large) in $G$, each of these subgroups can be lifted to a large nonopen subgroup of $G$, as in 2.3.

THEOREM 2.5. Let $G$ be a compact totally disconnected nonmetrizable group. Then:

(1) $G$ has $2^{|a|}$ large nonopen subgroups.

(2) Every neighborhood of e contains a large nonopen subgroup of $G$.

(3) If $G$ is Abelian, $G$ has $2^{|G|}$ discontinuous torsion characters.

Proof. Let $\mathscr{B}$ be any collection of open normal subgroups of $G$, such that $\cap \mathscr{B}=e$. By 2.2, and the obvious generalization of Theorem 1.2 of [6], $|\mathscr{B}|=w(G)$. Since $\mathscr{B}$ is uncountable, there must be some integer $n$ and some subcollection $\mathscr{U}$ of $\mathscr{B}$ such that $|\mathscr{U}|=w(G)$ and $|G / U| \leqq n$ for all $U \in \mathscr{Q}$. Theorem 2.4 therefore applies, and, since $2^{w(G)}=|G|$ (see [4]), statement (1) follows.

(2) follows from the proof of 1.9 ; (3) follows from 1.7.

CoROLlaRY 2.6. Let $G$ be a locally compact group such that $G / C_{G}$ is compact and nonmetrizable. Then $G$ has at least $2^{c}$ large nonopen subgroups. If $G$ is Abelian, it has at least $2^{c}$ discontinuous torsion characters. 


\section{Examples and questions.}

3.1. Let $G=\prod_{n=1}^{\infty} Z\left(2^{n}\right)$, where $Z(m)$ denotes the additive group of integers modulo $m$. Let

$$
J_{1}=\{0\}, \cdots, J_{n}=\left\{0,2, \cdots, 2^{n}-2\right\} \in Z\left(2^{n}\right), \cdots, \text { and } J=\prod_{n=1}^{\infty} J_{n} .
$$

Then $G / J=\{0,1\}^{\omega}$, so $G$ has $2^{c}$ large nonopen subgroups, by 2.3 and Case (1) of 2.4. (Alternately, $G$ has $2^{c}$ discontinuous characters of order 2.)

Note: This $G$ contains an infinite compact subgroup with no large subgroups, for the monothetic group $\Delta_{2}$ of dyadic integers is topologically isomorphic to the projective limit of the groups $Z\left(2^{n}\right)$. (Let $\Lambda_{n}=\left\{x \in \Delta_{2}: x_{k}=0,0 \leqq k<n\right\}$, as in 10.4 of [3]. According to p. 25 of [9], $\Delta_{2}$ is an inverse limit of the quotient groups $\Delta_{2} / A_{n}$. It is clear that $\Delta_{2} / \Lambda_{n} \cong Z\left(2^{n}\right)$. See also $6.13-6.14$ of [3] and the references noted in 1.5 , above.)

3.2. Let $G=\prod_{n=5}^{\infty} S_{n}, J=\prod_{n=5}^{\infty} A_{n}$, where $S_{n}$ is the symmetric group, and $A_{n}$ is the alternating group, on $n$ letters. As in the previous example, $G$ has $2^{c}$ large nonopen subgroups. Its subgroup $J$ has no large nonopen subgroups, for if $L$ is a subgroup of $J$ with $J^{(k)} \subset L$, we have (assuming $k \geqq 4$ )

$$
L \supset\{e\}^{k} \times \prod_{n=k+1}^{\infty} A_{n},
$$

which is open in $J$.

3.3. Suppose $G$ is an infinite, compact, metrizable, non-Abelian torsion group. (By 4.5 of [5], $G$ is totally disconnected.) Question: Does $G$ have any large nonopen subgroups?

3.4. Let $G=\prod_{n=1}^{\infty} G_{n}$, where each $G_{n}$ is a finite simple group and $\lim _{n \rightarrow \infty}\left|G_{n}\right|=\infty$. Question: Can $G$ have a large nonopen subgroup?

[The author conjectures that $G_{n}^{(k)} \neq\{e\}$ for $n$ sufficiently larger than $k$; this would imply that the answer to the question is in the negative, as in 3.2. If the conjecture should prove to be false, we would then have a special case of 3.3 , wherein $G$ would be semisimple and of bounded order.]

3.5. In order for the conclusion of 1.7 not to hold, $G$ must be noncompact, have infinitely many open subgroups, only finitely many 
large subgroups, and at least one large nonopen subgroup. The groups we have seen in this paper either have no large nonopen subgroups at all, or else $2^{2 r}$ such subgroups, where $r$ is the cardinal number of some infinite collection of large open subgroups. Question: Are there other possibilities?

\section{REFERENCES}

1. G. Birkhoff and S. MacLane, A Survey of Modern Algebra: revised edition. Macmillan Co., New York, 1953.

2. L. Gilman and M. Jerison, Rings of Continuous Functions, D. Van Nostrand Co., Princeton, 1960.

3. E. Hewitt and K. A. Ross, Abstract Harmonic Analysis I, Springer-Verlag, Heidelberg, 1963.

4. A. Hulanicki, On cardinal numbers related with locally compact groups, Bull. Acad. Polon. Sci. Math. Astr. Phys., VI-2 (1958), 67-70.

5. R. Iltis, Some algebraic structure in the dual of a compact group, Canad. J. Math., 20 (1968), 1499-1510.

6. S. Kakutani, On cardinal numbers related with a compact Abelian group, Proc. Imp. Acad. Tokyo, 19 (1943), 366-372.

7. J. Mycielski, Some properties of connected compact groups, Colloq. Math., 5 (1958), 162-166.

8. N. Th. Varopoulos, A theorem on the continuity of homomorphisms of locally compact groups, Proc. Cambridge Philos. Soc., 60 (1964), 449-463.

9. A. Weil, L'integration dans les Groups Topologiques et ses Applications, Actualités Sci. et Ind. 869-1145, Hermann \& Cie., Paris, 1951.

Received October 4, 1971 and in revised form August 30, 1972.

INDIANA UNIVERSITY NORTHWEST 



\section{PACIFIC JOURNAL OF MATHEMATICS}

\section{EDITORS}

\section{H. SAMELSON}

Stanford University

Stanford, California 94305

C. R. Новву

University of Washington Seattle, Washington 98105

\section{J. DuGundJI}

Department of Mathematics University of Southern California Los Angeles, California 90007

RICHARD ARENS

University of California Los Angeles, California 90024

\section{ASSOCIATE EDITORS}
E. F. BECKENBACH
B. H. NeumanN
F. WOLF
K. YoSHIDA

\section{SUPPORTING INSTITUTIONS}

\author{
UNIVERSITY OF BRITISH COLUMBIA \\ CALIFORNIA INSTITUTE OF TECHNOLOGY \\ UNIVERSITY OF CALIFORNIA \\ MONTANA STATE UNIVERSITY \\ UNIVERSITY OF NEVADA \\ NEW MEXICO STATE UNIVERSITY \\ OREGON STATE UNIVERSITY \\ UNIVERSITY OF OREGON \\ OSAKA UNIVERSITY
}

\author{
UNIVERSITY OF SOUTHERN CALIFORNIA \\ STANFORD UNIVERSITY \\ UNIVERSITY OF TOKYO \\ UNIVERSITY OF UTAH \\ WASHINGTON STATE UNIVERSITY \\ UNIVERSITY OF WASHINGTON \\ $*{ }^{*}$
AMERICAN MATHEMATICAL SOCIETY
NAVAL WEAPONS CENTER
}

The Supporting Institutions listed above contribute to the cost of publication of this Journal, but they are not owners or publishers and have no responsibility for its content or policies.

Mathematical papers intended for publication in the Pacific Journal of Mathematics should be in typed form or offset-reproduced, (not dittoed), double spaced with large margins. Underline Greek letters in red, German in green, and script in blue. The first paragraph or two must be capable of being used separately as a synopsis of the entire paper. The editorial "we" must not be used in the synopsis, and items of the bibliography should not be cited there unless absolutely necessary, in which case they must be identified by author and Journal, rather than by item number. Manuscripts, in duplicate if possible, may be sent to any one of the four editors. Please classify according to the scheme of Math. Rev. Index to Vol, 39. All other communications to the editors should be addressed to the managing editor, Richard Arens, University of California, Los Angeles, California, 90024.

50 reprints are provided free for each article; additional copies may be obtained at cost in multiples of 50 .

The Pacific Journal of Mathematics is issued monthly as of January 1966. Regular subscription rate: $\$ 48.00$ a year (6 Vols., 12 issues). Special rate: $\$ 24.00$ a year to individual members of supporting institutions.

Subscriptions, orders for back numbers, and changes of address should be sent to Pacific Journal of Mathematics, 103 Highland Boulevard, Berkeley, California, 94708.

PUBLISHED BY PACIFIC JOURNAL OF MATHEMATICS, A NON-PROFIT CORPORATION

Printed at Kokusai Bunken Insatsusha (International Academic Printing Co., Ltd.), 270, 3-chome Totsuka-cho, Shinjuku-ku, Tokyo 160, Japan. 


\section{Pacific Journal of Mathematics}

\section{Vol. 44, No. $2 \quad$ June, 1973}

Tsuyoshi Andô, Closed range theorems for convex sets and linear liftings . . . . . . 393

Richard David Bourgin, Conically bounded sets in Banach spaces . . . . . . . . . 411

Robert Jay Buck, Hausdorff dimensions for compact sets in $R^{n} \ldots \ldots \ldots \ldots \ldots \ldots . \ldots 421$

Henry Cheng, A constructive Riemann mapping theorem ................ 435

David Fleming Dawson, Summability of subsequences and stretchings of

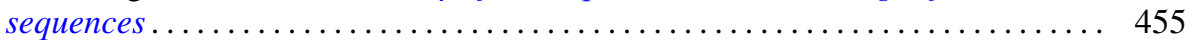

William Thomas Eaton, A two sided approximation theorem for 2-spheres ....... 461

Jay Paul Fillmore and John Herman Scheuneman, Fundamental groups of compact complete locally affine complex surfaces ....................... 487

Avner Friedman, Bounded entire solutions of elliptic equations . . . . . . . . . . . 497

Ronald Francis Gariepy, Multiplicity and the area of an $(n-1)$ continuous

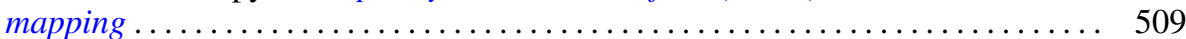

Andrew M. W. Glass, Archimedean extensions of directed interpolation groups . . . . 515

Morisuke Hasumi, Extreme points and unicity of extremum problems in $H^{1}$ on

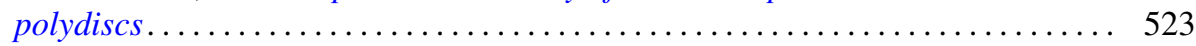

Trevor Ongley Hawkes, On the Fitting length of a soluble linear group . . . . . . 537

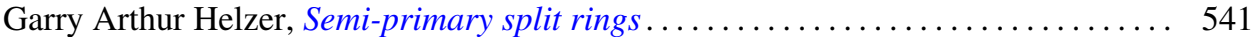

Melvin Hochster, Expanded radical ideals and semiregular ideals . . . . . . . . . 553

Keizō Kikuchi, Starlike and convex mappings in several complex variables . . . . . . 569

Charles Philip Lanski, On the relationship of a ring and the subring generated by its

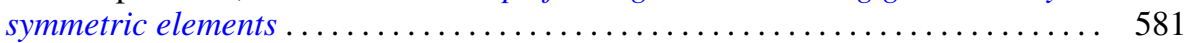

Jimmie Don Lawson, Intrinsic topologies in topological lattices and semilattices ........................................... 593

Roy Bruce Levow, Counterexamples to conjectures of Ryser and de Oliveira ...... 603

Arthur Larry Lieberman, Some representations of the automorphism group of an infinite continuous homogeneous measure algebra ..........

William George McArthur, $G_{\delta}$-diagonals and metrization theorems $\ldots .$.

James Murdoch McPherson, Wild arcs in three-space. II. An invariant of

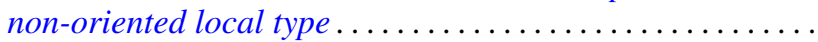

H. Millington and Maurice Sion, Inverse systems of group-valued measures ...

C. Edward Moore, Concrete semispaces and lexicographic separation of convex

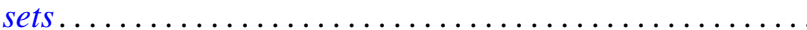

Jingyal Pak, Actions of torus $T^{n}$ on $(n+1)$-manifolds $M^{n+1}$.

Merrell Lee Patrick, Extensions of inequalities of the Laguerre and Turán type . . . . 675

Harold L. Peterson, Jr., Discontinuous characters and subgroups of finite index. . . . 683

S. P. Philipp, Abel summability of conjugate integrals . . . . . . . . . . . . . 693

R. B. Quintana and Charles R. B. Wright, On groups of exponent four satisfying an

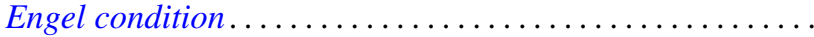

Marlon C. Rayburn, On Hausdorff compactifications. . . . . . . . . .

Martin G. Ribe, Necessary convexity conditions for the Hahn-Banach theorem in

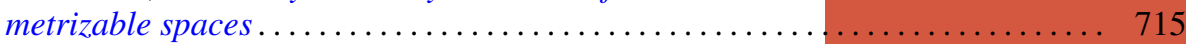

Ryōtarō Satō, On decomposition of transformations in infinite measure spaces .... 733

Peter Drummond Taylor, Subgradients of a convex function obtained from a

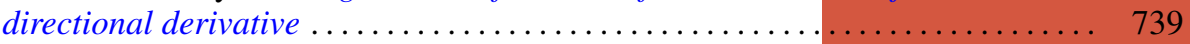

James William Thomas, A bifurcation theorem for $k$-set contractions . . . . . . . . 749 Clifford Edward Weil, A topological lemma and applications to real functions . . . . 757

Stephen Andrew Williams, A nonlinear elliptic boundary value problem . . ....... 767

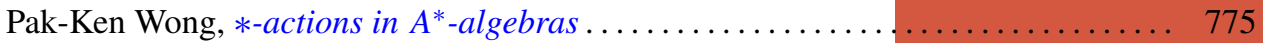

